\title{
For the mind's eye the world is two-dimensional
}

\author{
JANEK S. LOBMAIER \\ University of Zurich, Zurich, Switzerland \\ and University of St. Andrews, St. Andrews, Scotland \\ Fred W. Mast \\ University of Zurich, Zurich, Switzerland \\ and University of Bern, Bern, Switzerland \\ AND \\ HeIKo HeChT \\ Johannes Gutenberg-Universität Mainz, Mainz, Germany
}

\begin{abstract}
The nature of visual mental images is a topic that has puzzled neuroscientists, psychologists, and philosophers alike. On the one hand, mental images might preserve the 3-D properties of our perceptual world. On the other hand, they might be akin to 2-D pictures, such as photographs, paintings, or drawings. In the present study, 16 observers judged where real objects (Experiment 1) or photographs thereof (Experiment 2) were pointing. Both experiments contained a perception condition and an imagery condition. In Experiment 1, there was a significant difference between the pointing errors in the perception and the imagery conditions, whereas there was no such difference in Experiment 2. In imagined objects, actual photographs, and imagined photographs, the direction in which the objects pointed followed the observer, regardless of his or her vantage point. The results from this study extend the rotation effect, typically found in pictures, to the domain of mental imagery. We found the rotation effect in pictures and mental images alike, but not in direct perception of 3-D objects; thus, we provide evidence that mental images share main characteristics of 2-D pictures.
\end{abstract}

The psychological nature of visual mental images has remained an elusive topic (Klein et al., 2004; Kosslyn \& Thompson, 2003; Pylyshyn, 2003). Our perceptual world is 3-D in a very fundamental sense, regardless of whether one draws upon constructivist theories (Helmholtz, 1971; Rock, 1983) or on notions of direct visual perception (Gibson, 1979). Mental images, however, are much more difficult to gauge. On the one hand, it is possible that mental images embody the very $3-\mathrm{D}$ structure that is relevant for our phenomenal world. One could liken such 3-D representations to stereoscopic images or to simulacra of the objects they represent. On the other hand, mental images could rely on a representational format that is inherently 2-D, thereby resembling photographs, paintings, or drawings. Which of these two fundamentally different alternatives constitutes the representational nature of visual mental images has not yet been resolved.

We addressed the representational nature of mental images by making use of what we take to be a decisive attribute that distinguishes pictures from objects: the rotation effect (Cutting, 1987, 1988; Goldstein, 1979, 1987, 1988). Leonardo da Vinci's Mona Lisa has often been used to illustrate the rotation effect, which refers to the phenomenon that an observer who changes the vantage point in front of the portrait has the striking experience that Mona Lisa's gaze is following him or her. The rotation effect is most noticeable in portraits, but it can be found for a large variety of depicted objects (Goldstein, 1979). In 3-D objects, however, the rotation effect is absent. The rotation effect has been explained in various ways. For instance, the visual system has been thought to compensate for changes in the retinal image caused by observer displacement (for details, see Pirenne, 1970). Others have described the percept on the array-specific interpretation of the retinal image (e.g., Goldstein, 1987). Since the optic array does not change with observer displacement (it is only distorted), the percept remains unchanged. In contrast, the percept of 3-D objects will change with observer displacement. We took advantage of this anisotropy of the rotation effect's being present in 3-D, but not in 2-D, objects to explore the representational nature of imagined objects. We did so according to the rationale that if an imagined object shares its representational format with a percept of a real 3-D object that we behold, the rotation effect should not apply to imagined objects. If, however, imagined objects are pictorial in nature, the rotation effect should be found for imagined objects.

The view of actual objects in perceptual space changes as the observer moves around in a stable environment (Koenderink \& van Doorn, 2003; Koenderink, van Doorn,

F.W. Mast, fred.mast@psy.unibe.ch 
Kappers, \& Todd, 2004). The vista they reveal is directly yoked to the observer's vantage point. Unlike this perceptual space of the visual environment, pictorial space is largely independent of the observer's viewing position. Objects in pictorial space follow the observer because their orientation is defined with respect to the canvas, but not with respect to the observer's viewpoint (see, e.g., Hecht, Schwartz, \& Atherton, 2003). On the basis of this disparity between perceptual and pictorial space, we made the following predictions. A physical displacement of the observer should strongly affect the perceived orientation of a real object with respect to his or her own position in space. However, no such difference in perceived orientation should be found before and after an observer's displacement in front of the photograph of the same object. If the observer is asked to perform the same judgments on a mental image of the object, before and after being displaced, we can deduce from the judged orientations whether the imagined object embodies the 3-D properties of a real object or whether it behaves like a picture thereof.

In the present study, we thus compared the size of the rotation effect in physical, pictorial, and merely imagined objects. In all cases, the observer's vantage point was changed from the 0-point straight in front of the object to positions toward the left or the right. If a rotation effect was present, judgments should differ from the target point as a function of observer displacement to either side.

In the first experiment, real objects were used. In the perception condition, observers saw an actual bust, a cubic object, or a cuboid object. In the imagery condition, observers imagined the actual objects. In the second experiment, observers saw photographs of the same objects as in Experiment 1 (perception condition) or merely imagined the corresponding photographs. In all cases, the observers were asked to judge where the object was pointing with respect to their own position in space. Note that observers are capable of performing such a task with satisfactory accuracy on the basis of pictures of faces and eyes (Cline, 1967; Schwaninger, Lobmaier, \& Fischer, 2005). Also, previous research has shown that people are indeed able to imagine an object in its genuine size (Kosslyn, Thompson, Kim, \& Alpert, 1995); thus, the would-be visual angle of the imagined object can be taken to correspond to the visual angle when the object is perceptually present.

\section{METHOD}

\section{Participants}

Sixteen healthy participants ( 8 women, 8 men), ranging in age from 22 to 34 years (mean, 27 years), took part in this study. Two participants reported being left-handed, and all reported normal or corrected-to-normal vision. The participants underwent the 3-D experiment (Experiment 1) and the 2-D experiment (Experiment 2) in one session. They all gave written, informed consent and received payment for their participation. The participants were treated according to the declaration of Helsinki.

\section{Stimuli and Experimental Procedure}

The experiments were run in a well-lit room. All the participants underwent both experiments, Experiment 1 with 3-D objects and Experiment 2 with 2-D pictures of these objects. The order of Ex- periments 1 and 2 was counterbalanced across participants. Both the 3-D and 2-D experiments contained three blocks (bust, cube, cuboid), and each block contained a perception and an imagery condition. The order of blocks was counterbalanced across participants. The imagery condition was always performed before the perception condition. This was done (1) to ensure that all the participants had approximately the same amount of time to encode the stimuli in order to form mental representations and (2) because performing the perception task first might have influenced the performance in the imagery condition.

Experiment 1:3-D objects. At one end of the room, one of three different objects was placed on a stand. A naturalistic bust, a cuboid $(15 \times 15 \times 35.5 \mathrm{~cm})$, and a cubic box $(20 \times 20 \times 20 \mathrm{~cm})$ were used. These objects were placed on a tripod at eye level. The participants were seated on a height-adjustable chair at a distance of $1.5 \mathrm{~m}$ away from the object. A headrest ensured that viewing distance was kept constant. The objects subtended a visual angle of $5.7^{\circ}$ (bust and cuboid) or $7.6^{\circ}$ (cube). The participants viewed each object from seven different viewpoints. The objects remained at the same location at all times, but the participants were seated at different locations defined by an orbital line around the object: $0^{\circ}$ (straight ahead of the object) and $5^{\circ}, 10^{\circ}$, and $15^{\circ}$ to the left or right of the object, keeping the distance to the object $(1.5 \mathrm{~m})$ constant. The objects were turned $5^{\circ}$ out of the frontoparallel plane to prevent the objects from facing the observer in the $0^{\circ}$ position. This $5^{\circ}$ turn was kept constant in all locations.

Before the experiment proper, all three objects were presented to the participants, and they were asked to walk around them in order to avoid a view-biased representation of the object. The imagery condition was preceded by an encoding phase. In this encoding phase, the object was presented to the participants while they sat in the $0^{\circ}$ position. The participants were asked to carefully examine and memorize the object. The experimenter then occluded the object with a screen, and the participants were required to visualize the object using mental imagery. After a short while, the object was uncovered again, and the participants could correct their visual image. This procedure was repeated at least five times. During the following imagery trials, the object was removed. The participants were instructed to vividly imagine that the object was still present on the stand just as they had seen it, in its original size and position. They were asked to indicate on an aluminium bar where the object was pointing while vividly imagining the object in its original location (see Figure 1). The (empty) stand remained at a constant location in the room while the participants were moved to different viewing angles, as described above. All imagery trials were performed in one sequence, without any further viewing of the object. In the perception condition, the object was physically present. In both conditions, the observers judged where the object was pointing by marking a location (target point) on an aluminium bar (1.4 m long), which was situated $30 \mathrm{~cm}$ in front of the participants. The participants moved a slider on this bar to the exact position at which they perceived that an extension of the object's axis (gaze line of the bust or elongated axis of the cube/cuboid) would intersect with the bar. On the rear side of the bar, a tape measure was attached, which was visible only to the experimenter. Judgments were recorded by registering the positions of the slider. Before each trial, the slider was set to one extreme position on the bar, alternating between the right and left ends. In both conditions, the participants were relocated to a new position after each trial. While they were relocated, the object was occluded by the screen. Each participant underwent 42 trials ( 7 viewing angles $X$ 3 objects $\times 2$ conditions). The order of viewing angles was counterbalanced across participants.

Experiment 2: 2-D pictures. The apparatus, task, and procedure were the same as those in the 3 -D objects condition, except that photographs of the objects were used instead of the 3-D objects. Each photograph was taken from the $0^{\circ}$ position of the viewer. Thus, the constant $5^{\circ}$ shift out of the frontoparallel plane was the same as that in the 3-D condition. The photograph was then placed at exactly the same position (i.e., same distance, same height) as the 3-D objects. 


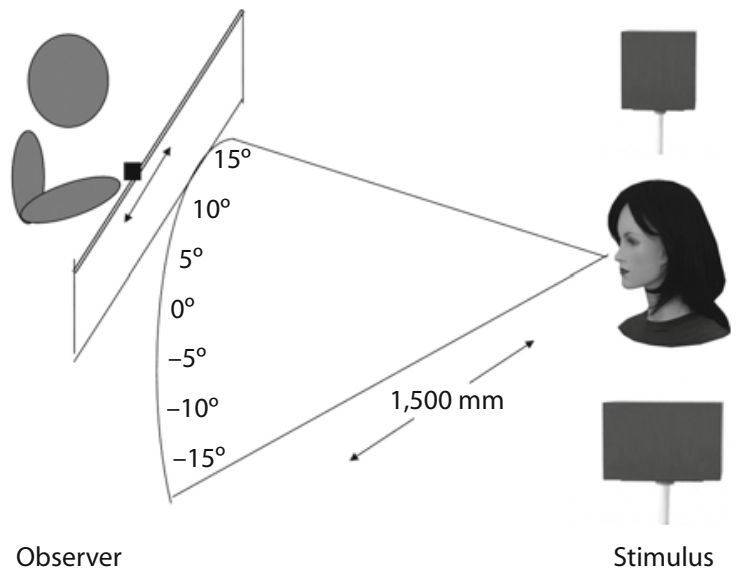

Figure 1. Experimental setup: In the perception condition, participants consecutively saw a bust, a cube, or a cuboid (Experiment 1) or photographs thereof (Experiment 2) and judged on a slider where they perceived the object to point. In the imagery conditions, no object/picture was presented, and participants made their judgments using visual mental imagery.

The only difference between Experiments 1 and 2 was the dimensionality of the stimuli.

\section{Data Analyses}

For each measurement, the rotation effect was measured as the deviation between pointing direction of the veridical target and the observers' adjustment. The deviation was then transformed into angles (degrees). Any systematic deviation between the veridical pointing direction and the observers' judgment would imply a rotation effect. No difference between judgment and veridical pointing direction would mean no rotation effect. Separately for the 3-D and 2 -D experiments, these values were analyzed in a 3 (head, cuboid, cube) $\times 2$ (imagery, perception) $\times 7$ (viewing angle) repeated measures ANOVA.

\section{RESULTS}

\section{Experiment 1: 3-D Objects}

For 3-D objects, the repeated measures ANOVA revealed a significant main effect of viewing angle $[F(6,90)=$ $\left.8.03, M S_{\mathrm{e}}=290.424, p<.01, \eta^{2}=.349\right]$. Object type $\left[F(2,30)=0.18, M S_{\mathrm{e}}=82.979, p=.83, \eta^{2}=.012\right]$ and experimental condition $\left[F(1,15)=1.60, M S_{\mathrm{e}}=67.798\right.$, $\left.p=.225, \eta^{2}=.097\right]$ were not significant. However, the two-way interaction of experimental condition and viewing angle was highly significant, indicating a rotation effect for imagined objects, but not for physical objects $\left[F(6,90)=28.48, M S_{\mathrm{e}}=105.696, p<.001, \eta^{2}=.652\right.$; see Figure 2A]. Post hoc two-way ANOVAs including the factors of object and viewing angle, calculated separately for the imagery and perception conditions, revealed that the main effect of viewing angle was significant only in the imagery condition $\left[F(6,90)=22.06, M S_{\mathrm{e}}=257.902\right.$, $\left.p<.001, \eta^{2}=.595\right]$. In the perception condition there was no effect of viewing angle. This implies that there was a rotation effect only for imagined objects, but not when the objects were physically present. All other interactions did not reach statistical significance (all $F \mathrm{~s}<1.02$, $p \mathrm{~s}>.431)$.

\section{Experiment 2: 2-D Pictures}

For 2-D pictures, the repeated measures three-way ANOVA revealed a significant effect of viewing angle $\left[n=16 ; F(6,90)=64.48, M S_{\mathrm{e}}=196.996, p<.001\right.$, $\eta^{2}=.81$, indicating a substantial rotation effect (see Figure 2B). No other main effects (all $F \mathrm{~s}<0.25, p \mathrm{~s}>.653$ ) and none of the interactions (all $F \mathrm{~s}<2.68, p \mathrm{~s}>.09$ ) reached statistical significance, indicating no difference between imagery and perception of 2-D pictures. To test for different patterns across 3-D objects and 2-D pictures, we conducted a four-way ANOVA including data from both experiments. This analysis revealed no main effect of experiment $\left[F(1,15)=1.72, M S_{\mathrm{e}}=208.346, p=.209\right.$, $\left.\eta^{2}=.103\right]$; however, the two-way experiment $\times$ viewing angle interaction $\left[F(6,90)=21.95, M S_{\mathrm{e}}=24.442\right.$, $\left.p<.001, \eta^{2}=.594\right]$ and the three-way experiment $\times$ viewing angle $\times$ condition interaction $[F(6,90)=37.07$, $\left.M S_{\mathrm{e}}=17.698, p<.001, \eta^{2}=.712\right]$ reached statistical significance. No other interactions containing the factor of experiment turned out to be significant (all $F_{\mathrm{s}}<1.94$, ps $>.161)$.

\section{DISCUSSION}

In the present study, we used the rotation effect to explore the spatial properties of mental visual images of objects. For perceived 3-D objects, there was no rotation effect. However, when these objects were imagined, they showed the very characteristics that are pertinent to the perception of 2-D pictures. The direction in which the imagined objects pointed followed the observer, regardless of his or her vantage point. Hence, mental images of extended objects seem to lose an important 3-D spatial property. For 2-D pictures, the classic rotation effect was found to the same extent in all perception and imagery conditions; there was no appreciable difference between directly viewing a photograph of an object and imagining it.

Although a mental image of an object may preserve 3-D information, it preserves it in the same fashion as does a 2-D picture. When performing a mental rotation task of a cube, we are, of course, able to figure out how it should appear when rotated. However, we suggest that this spatial ability cannot be fully based on a mental representation that maintains 3-D properties. Our findings support the pictorial nature of mental images (Kosslyn, Ball, \& Reiser, 1978; Kosslyn \& Thompson, 2003; Kosslyn et al., 1995), and they are in line with picture theories of object recognition (Bülthoff, Edelman, \& Tarr, 1995). The rotation effect in all imagery conditions and its entire absence in the face of the physical objects demonstrates that participants rely on a depictive format of representation when they judge the orientation of imagined objects. Even though phenomenal experience suggests that mental images may appear truly 3-D (see, e.g., Blanz, Tarr, \& Bülthoff, 1999), their orientation in space with respect to the observer is not adequately represented.

Three potential alternative explanations might be summoned to challenge this interpretation of the present data. First, Blanz et al. (1999) presented data suggesting that imagined objects have a preferred orientation. This pre- 
A

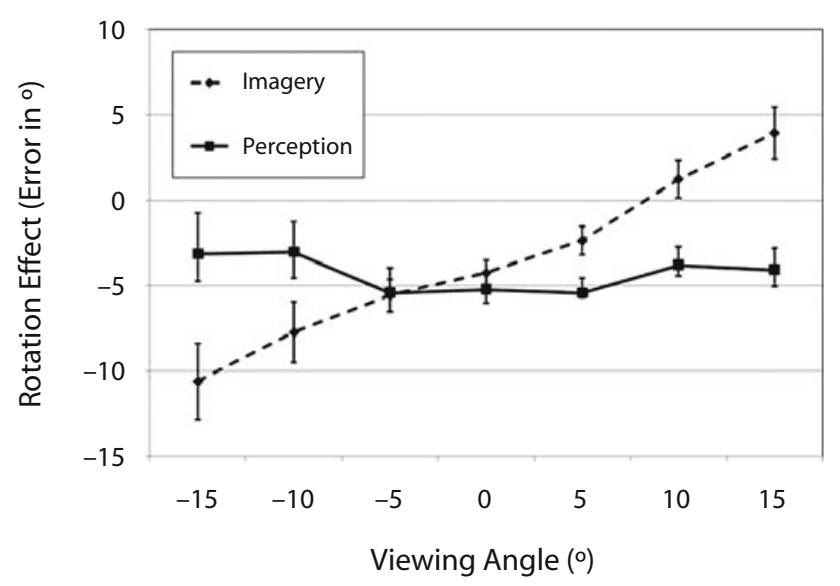

B

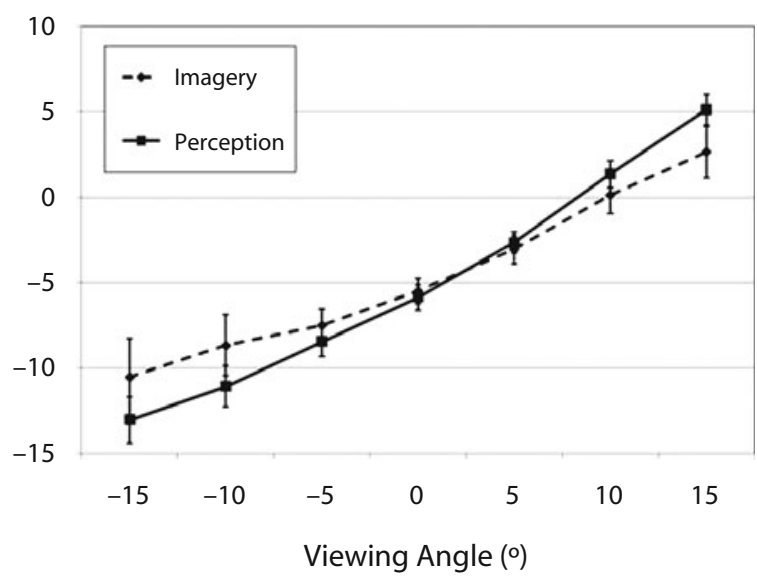

Figure 2. (A) Real objects and their mental images: Average errors that were produced when participants were asked to judge where an object was pointing, pooled across objects. Diamonds depict imagery conditions; squares depict perception conditions. Error bars depict SEMs. (B) Photographs and their mental images: Average pointing errors on the basis of photographs of objects versus imagined photographs, pooled across objects. Diamonds depict imagery conditions; squares depict photo-perception conditions. Error bars depict $S E M$ s.

ferred orientation may have coincided with the $0^{\circ}$ view of the objects in the present study and may have forced the pointing errors. However, such an orientation preference should have influenced the perceived pointing direction of real objects as well. If one came up with a convincing case for why preferred orientation should affect images but not real objects, this notion could be used to explain our results. At present, we do not see how such a case could be made.

The second explanation is more radical. It suggests that observers might have been unable to follow instructions. They could have yoked the mental image to their body position and moved the representation along with their body, despite instructions to the opposite. On theoretical grounds, it may be arguable that the failure to follow the instruction to leave the mental image of the object in place amounts to the rotation effect. The notion of a disembodied eye, always looking at a picture from the same place, implies that the instruction to reembody the eye is bound to fail. If participants were indeed unable to follow our instructions, the ultimate consequence would be that human beings are unable to perform spatial updating. There is, however, ample evidence suggesting that this is not the case (e.g., Loomis, Lippa, Golledge, \& Klatzky, 2002). Furthermore, if participants simply recalled the image as they encoded it, with no updating for position, this would result in the angular pointing error's being equal to the viewing angle. In general, the errors have smaller magnitudes than the viewing angles, and, importantly, the errors are similar to those for pictures.

Finally, it could be argued that participants had a preferred orientation for their mental images that may have interacted with their viewing position. Given that the results in the imagery condition for the 3-D objects revealed the same pattern as that in both the conditions in the 2-D experiment, this explanation seems unlikely. It will have to be the aim of further studies to exclude this possible explanation.

Attempts to move away from a picture theory of mental imagery toward a propositional notion claiming that to imagine is to simulate what would happen when looking at the physical object (Pylyshyn, 2002) do not seem to be justified in the face of our findings. Had the participants made the judgments on the basis of their knowledge about the appearance of the object, no rotation effect should have been observed during imagery. Hence, the participants did not reproduce in imagery where the object was pointing when they saw it during the encoding phase. The present data show that the discerned orientation of imagined objects is fundamentally different from the perceived orientation of physical objects, despite the fact that many spatial properties are shared between imagery and perception (Kosslyn et al., 1978; Kosslyn \& Thompson, 2003; Kosslyn et al., 1995). This conclusion has consequences for our understanding of mental imagery at large. We propose that the ability to mentally rotate objects (Shepard \& Metzler, 1988) has to be reinterpreted as a capability to manipulate pictorial representations of 3-D objects, rather than being an ability to represent the objects threedimensionally (Pinker, 1980). While our findings show that the directions in which imagined objects appear to point are picture-like, this does not entail that 3-D information is entirely lost in mental representations. In some circumstances, it is still possible for images to be coded as 3-D representations (e.g., Kozhevnikov \& Hegarty, 2001). It is the relation to the observer that might be compromised in this representation, while the 3-D structure of the object remains intact. Path finding and spatial updating of our own position when navigating in a momentarily invisible world requires a 3-D representation (Loomis et al., 2002). For these tasks, however, it is sufficient to know where in the world the targets are located. Amorim, Glas- 
auer, Corpinot, and Berthoz (1997) had observers use a physical model to re-create the orientation of a sculpted letter that they had seen previously. These re-creations betrayed some systematic errors, but all in all the observers were rather accurate, and the errors were robust over a displacement of the blindfolded observer. This evidence for a general ability to spatially update the orientation of an object from an object-centered, as well as from a trajectory-based, frame of reference has to be reconciled with our findings. The task of re-creating the orientation of an object might be fundamentally different from discerning the pointing direction of the object in the observer's space. This possibility certainly warrants further investigation. Specifically, finding out the circumstances in which mental images are encoded as 3-D representations and in which cases there are represented as 2-D will have to be the aim of future studies.

Our data suggest that visually imagined objects may be represented in the right place but they are not oriented in the proper direction. Mental images rely on a depictive format of representation and, thus, share similarities with 2-D pictures. We conclude that seeing with the mind's eye is 2-D and, therefore, differs fundamentally from visual perception.

\section{AUTHOR NOTE}

This work was supported by a grant from the University of Zurich and by the Swiss National Science Foundation. We are grateful to Iwana Staedeli for her help in collecting the data. We thank John Kennedy, Hal Sedgwick, and Jack Loomis for insightful discussions of the rotation effect. Correspondence concerning this article should be addressed to F. W. Mast, Department of Psychology, University of Bern, Muesmattstr. 45, CH-3000 Bern, Switzerland (e-mail: fred.mast@psy.unibe.ch).

\section{REFERENCES}

Amorim, M.-A., Glasauer, S., Corpinot, K., \& Berthoz, A. (1997). Updating an object's orientation and location during nonvisual navigation: A comparison between two processing modes. Perception \& Psychophysics, 59, 404-418.

Blanz, V., Tarr, M. J., \& Bülthoff, H. H. (1999). What object attributes determine canonical views? Perception, 28, 575-599.

Bülthoff, H. H., Edelman, S. Y., \& Tarr, M. J. (1995). How are 3-D objects represented in the brain? Cerebral Cortex, 5, 247-260.

Cline, M. G. (1967). The perception of where a person is looking. American Journal of Psychology, 80, 41-50.

CutTing, J. E. (1987). Rigidity in cinema seen from the front row, side aisle. Journal of Experimental Psychology: Human Perception \& Performance, 13, 323-334.

CutTing, J. E. (1988). Affine distortions of pictorial space: Some predictions for Goldstein (1987) that La Gournerie (1859) might have made. Journal of Experimental Psychology: Human Perception \& Performance, 14, 305-311.

Gibson, J. J. (1979). The ecological approach to visual perception. Hillsdale, NJ: Erlbaum.

Goldstein, E. B. (1979). Rotation of objects in pictures viewed at an angle: Evidence for different properties of two types of pictorial space. Journal of Experimental Psychology: Human Perception \& Performance, 5, 78-87.

Goldstein, E. B. (1987). Spatial layout, orientation relative to the observer, and perceived projection in pictures viewed at an angle. Journal of Experimental Psychology: Human Perception \& Performance, 13, 256-266.

Goldstein, E. B. (1988). Geometry or not geometry? Perceived orientation and spatial layout in pictures viewed at an angle. Journal of Experimental Psychology: Human Perception \& Performance, 14, 312-314.

Hecht, H., Schwartz, R., \& Atherton, M. (Eds.) (2003). Looking into pictures: An interdisciplinary approach to pictorial space. Cambridge, MA: MIT Press.

HeLMHOLTZ, H. VON (1971). The origin and correct interpretation of our sense impressions. In R. Hahl (Ed.), Selected writings of Hermann von Helmholtz (pp. 501-512). Middletown, CT: Wesleyan University Press.

Klein, I., Dubois, J., Mangin, J.-F., Kherif, F., Flandin, G., Poline, J. B., ET AL. (2004). Retinotopic organization of visual mental images as revealed by functional magnetic resonance imaging. Cognitive Brain Research, 22, 26-31.

KoenderinK, J. J., \& van Doorn, A. J. (2003). Pictorial space. In H. Hecht, R. Schwartz, \& M. Atherton (Eds.), Looking into pictures: An interdisciplinary approach to pictorial space (pp. 239-299). Cambridge, MA: MIT Press.

Koenderink, J. J., van Doorn, A. J., Kappers, A. M., \& Todd, J. T. (2004). Pointing out of the picture. Perception, 33, 513-530.

Kosslyn, S. M., Ball, T. M., \& Reiser, B. J. (1978). Visual images preserve metric spatial information: Evidence from studies of image scanning. Journal of Experimental Psychology: Human Perception \& Performance, 4, 47-60.

KossLyn, S. M., \& ThOmpson, W. L. (2003). When is early visual cortex activated during visual mental imagery? Psychological Bulletin, 129, 723-746.

Kosslyn, S. M., Thompson, W. L., Kim, I. J., \& Alpert, N. M. (1995). Topographical representations of mental images in primary visual cortex. Nature, 378, 496-498.

Kozhevnikov, M., \& Hegarty, M. (2001). A dissociation between object manipulation spatial ability and spatial orientation ability. Memory \& Cognition, 29, 745-756.

LoOmis, J. M., LiPPA, Y., Golledge, R. G., \& KlatzKy, R. L. (2002). Spatial updating of locations specified by 3-D sound and spatial language. Journal of Experimental Psychology: Learning, Memory, \& Cognition, 28, 335-345.

PinkeR, S. (1980). Mental imagery and the third dimension. Journal of Experimental Psychology: General, 109, 354-371.

Pirenne, M. H. (1970). Optics, painting and photography. London: Cambridge University Press.

Pylyshyn, Z. W. (2002). Mental imagery: In search of a theory. Behavioral \& Brain Sciences, 25, 157-182; discussion, 182-237.

Pylyshyn, Z. W. (2003). Return of the mental image: Are there really pictures in the brain? Trends in Cognitive Sciences, 7, 113-118.

Rock, I. (1983). The logic of perception. Cambridge, MA: MIT Press.

Schwaninger, A., Lobmaier, J. S., \& Fischer, M. H. (2005). The inversion effect on gaze perception reflects processing of component information. Experimental Brain Research, 167, 49-55.

ShePARD, R., \& Metzler, D. (1988). Mental rotation: Effects of dimensionality of objects and type of task. Journal of Experimental Psychology: Human Perception \& Performance, 14, 3-11.

(Manuscript received June 8, 2009; revision accepted for publication October 14, 2009.) 